\title{
VARIETIES IN CAGES: A LITTLE ZOO OF ALGEBRAIC GEOMETRY
}

\author{
GABRIEL KATZ
}

\begin{abstract}
A $d^{\{n\}}$-cage $\mathrm{K}$ is the union of $n$ groups of hyperplanes in $\mathbb{P}^{n}$, each group containing $d$ members. The hyperplanes from the distinct groups are in general position, thus producing $d^{n}$ points where hyperplanes from all groups intersect. These points are called the nodes of $\mathrm{K}$. We study the combinatorics of nodes that impose independent conditions on the varieties $X \subset \mathbb{P}^{n}$ containing them. We prove that if $X$, given by homogeneous polynomials of degrees $\leq d$, contains the points from such a special set $\mathrm{A}$ of nodes, then it contains all the nodes of $\mathrm{K}$. Such a variety $X$ is very special: in particular, $X$ is a complete intersection.
\end{abstract}

\section{INTRODUCTION}

This paper is an extension and generalization of $[\mathrm{K}]$, which dealt with algebraic curves in plane cages, to algebraic varieties in the multidimensional cages (see Definition 1.1). In this text, we use the term "variety" as a synonym of "algebraic set".

Our tools are mostly combinatorial. They are based on some "Fubini's-flavored" versions of the Bésout Theorem in the spirit of [GHS], GHS1, and [GHS2]. Although the results of this paper fit well into the general framework of Cayley-Bacharach theorems (see [EGH], Theorem CB6), to apply this general machinery to the very special configurations of nodes from a given cage still requires some effort. So here we present a more direct and elementary argument, whose application is limited in scope, but geometrically transparent.

Let us consider two groups of lines in the plane (real or complex), each group comprising three lines. We call such a configuration $\mathrm{K}$ of six lines a $3 \times 3$-cage, or $3^{\{2\}}$-cage for short. We label the lines of the first group with red, and of the second group with blue. Assume that there are exactly 9 points where the blue lines intersect the red lines. We call them the nodes of the cage.

Our original motivation for studying the varieties in cages comes from the following classical result in the theory of plane cubic curves, a special case of Chasles' Theorem for a pair of cubic curves that meet at 9 points $\mathrm{Ch}$.

Theorem 1.1. (The Cage Theorem for Plane Cubics) Any plane cubic curve $\mathcal{C}$, passing through eight nodes of a $3 \times 3$-cage, will automatically pass through the ninth node.

Recall that Cage Theorem 1.1 reflects the associativity of the binary group operation "+" on an elliptic curve $\mathcal{C}$ (see Figure 1).

Definition 1.1. $A d^{\{n\}}$-cage $\mathrm{K}$ is a configuration of $n$ distinctly colored groups of d hyperplanes each (the entire hyperplane configuration $\mathrm{K}$ consists of $n d$ hyperplanes) located in the 


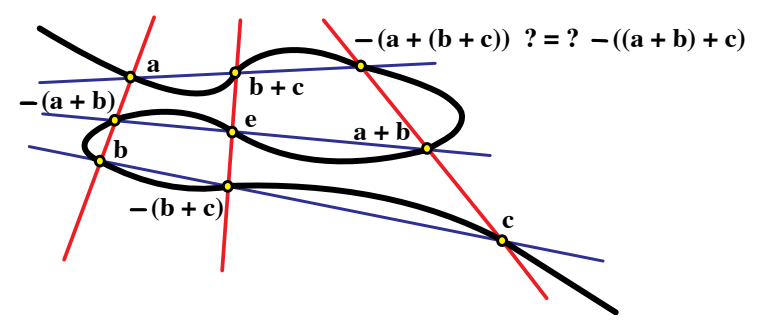

Figure 1. Cage Theorem 1.1 helps to validate the associativity of the group operation on a nonsingular cubic curve; $e$ denotes the neutral element.

$n$-space (projective or affine) in such a way, that $\mathrm{K}$ generates exactly $d^{n}$ points where the hyperplanes of all $n$ distinct colors $\alpha_{1}, \ldots, \alpha_{n}$ intersect transversally. (It follows that any $n$-tuple of distinctly colored hyperplanes are in general position in the ambient $n$-space.) These points are called the nodes of the cage.

Hyperplanes in $\mathbb{P}^{n}$ form a dual projective space $\mathbb{P}^{n *}$, the space of linear homogeneous functions, considered up to proportionality. Therefore $d$ hyperplanes of the same color from a $d^{\{n\}}$-cage $\mathrm{K} \subset \mathbb{P}^{n}$ represent an unordered configuration of $d$ points in $\mathbb{P}^{n *}$, a point in the symmetric product $\operatorname{Sym}^{d}\left(\mathbb{P}^{n *}\right)$. So the color-ordered collection of $n$ such points from $\operatorname{Sym}^{d}\left(\mathbb{P}^{n *}\right)$ is a point of the space $\left(\operatorname{Sym}^{d}\left(\mathbb{P}^{n *}\right)\right)^{n}$. By Definition 1.1, any set of $n$ hyperplanes of distinct colors has a single intersection point. The requirement that some set of $n$ hyperplanes of distinct colors has multiple intersection points in $\mathbb{P}^{n}$ puts algebraic constraints on the coefficients of the $d n$ homogeneous linear polynomials (in $n+1$ variables) that define the hyperplanes. Similarly, the requirement in Definition 1.1 that all transversal $n$-colored intersections are distinct, and thus numbering $d^{n}$, produces a Zariski open set. Therefore, we get: 
Lemma 1.1. The $d^{\{n\}}$-cages form a Zariski open set $\mathcal{K}$ in the $\left(d n^{2}\right)$-dimensional space $\left(\operatorname{Sym}^{d}\left(\mathbb{P}^{n *}\right)\right)^{n}$. The group of projective transformations $\mathrm{PGL}_{\mathbb{A}}(n+1)$ acts naturally on $\left(\operatorname{Sym}^{d}\left(\mathbb{P}^{n *}\right)\right)^{n}$, and thus on the set $\mathcal{K}$.

The problem we address in this paper is to describe the varieties that contain all $d^{n}$ nodes of a given cage $\mathrm{K}$. It turns out, that every variety $V$, defined by polynomials of degrees $\leq d$ and containing the node set $\mathrm{N}$, is very special indeed. In particular, $V$ must be a complete intersection (see Definition 2.2 of the type $(\underbrace{d, \ldots, d}_{s})$, where $s=n-\operatorname{dim} V$. Furthermore, the requirements that a hypersurface of degree $\leq d$ will pass through the nodes of a $d^{\{n\}_{-}}$ cage are very much redundant. In this article, we describe the combinatorics of the nodes that impose independent constraints on the hypersurface in question. We call such maximal set A of "independent" nodes supra-simplicial (see Definition 2.1 and Figure 22). Crudely, the proportion of cardinalities $\frac{\# A}{\# N}$ declines as $\sim 1 / n$ ! with the growth of $d$.

We have mentioned already that some of our results share the flavor with a remarkable family of classical theorems of Algebraic Geometry (see [C], C1], DGO, EGH], and Theorem 1.2 below). These classical theorems operate within a much less restrictive environment than the one of the $d^{\{n\}}$-cages. However, the theorems about varieties in cages are more geometrical, transparent, and easy to state.

To provide a point of reference, let us describe briefly this family of classical results, known under the name "Cayley-Bacharach theorems".

Let $\mathbb{Z}_{+}$denote non-negative integers. Recall that the Hilbert functions $h_{X}: \mathbb{Z}_{+} \rightarrow \mathbb{Z}_{+}$ of a variety $X$ over a field $\mathbb{A}$ associates with a non-negative integer $k$ the dimension of the $k$-graded portion of the quotient ring $\mathbb{A}\left[x_{0}, \ldots, x_{N}\right] / \mathcal{I}_{X}$, where $\mathcal{I}_{X}$ denotes the zero ideal that defines $X$. The ring $\mathbb{A}\left[x_{0}, \ldots, x_{N}\right] / \mathcal{I}_{X}$ is called the coordinate ring of $X$.

Since the node set $\mathrm{N}$ of a $(d \times d)$-cage is the intersection locus of $d$ red and $d$ blue lines, Theorem 1.1 is a special case of the Cayley-Bacharach Theorem $([\mathrm{C}],[\mathrm{B}])$, stated below. For a complete intersection $X \subset \mathbb{P}^{2}$, Theorem 1.2 connects the Hilbert functions $h_{X}: \mathbb{Z}_{+} \rightarrow \mathbb{Z}_{+}, h_{X_{1}}: \mathbb{Z}_{+} \rightarrow \mathbb{Z}_{+}$, and $h_{X_{2}}: \mathbb{Z}_{+} \rightarrow \mathbb{Z}_{+}$of a finite set $X$, its subset $X_{1}$, and its complement $X_{2}:=X \backslash X_{1}$. Recall that, for a 0-dimensional variety $X$ and all sufficiently big $k, h_{X}(k)=|X|$, the cardinality of $X$.

Theorem 1.2. (Cayley-Bacharach) Let $\mathcal{D}$ and $\mathcal{E}$ be two projective plane curves of degrees $d$ and $e$, respectively, and let the finite set $X=\mathcal{D} \cap \mathcal{E}$ be a complete intersection in $\mathbb{P}^{2}$. Assume that $X$ is the disjoint union of two subsets, $X_{1}$ and $X_{2}$. Then for any $k \leq d+e-3$, the Hilbert functions $h_{X}, h_{X_{1}}$, and $h_{X_{2}}$ are related by the formula:

$$
h_{X}(k)-h_{X_{1}}(k)=\left|X_{2}\right|-h_{X_{2}}((d+e-3)-k) .
$$

The RHS of this formula describes the failure to impose independent constrains by the points of the set $X_{2}$ on the polynomials of degree $k$. So Cayley-Bacharach Theorem may be viewed as a duality claim; however, it does not compute explicitly each of the dual quantities in the formula above. In contrast, our Theorem 2.1 accomplishes this task, 
provided $X=X_{1} \amalg X_{2}$ are very special: namely, $X$ is the 0 -dimensional variety of nodes of a cage $\mathrm{K}$, and $X_{1} \subset X$ is a supra-simplicial set.

Theorem 1.2 admits a comprehensive generalization by Davis, Geramita, Orecchia [DGO], and by Geramita, Harita, Shin (see [GHS1, and especially [GHS2, Theorem 3.13). It is a "Fubini-type" theorem for the Hilbert function of a finite subset $X \subset \mathbb{P}^{n}$ that is contained in the union of a family of hypersurfaces $\left\{H_{i}\right\}_{1 \leq i \leq s}$, whose degrees $\left\{d_{i}\right\}$ add up to the degree of $X$. Under some subtle hypotheses that regulate the interaction between $X$ and the hypersurfaces $\left\{H_{i}\right\}_{1 \leq i \leq s}$ (they include the hypotheses " $X=\coprod_{i}\left(X \cap H_{i}\right)$ "), a nice formula for the Hilbert functions $\left\{h_{X \cap H_{i}}: \mathbb{Z}_{+} \rightarrow \mathbb{Z}\right\}_{1 \leq i \leq s}$ of $H_{i}$-slices of $X$ emerges:

$$
h_{X}(k)=h_{X \cap H_{1}}(k)+h_{X \cap H_{2}}\left(k-d_{1}\right)+\cdots+h_{X \cap H_{s}}\left(k-\left(d_{1}+\cdots+d_{s-1}\right)\right) .
$$

A clear beautiful overview of the research, centered on the Cayley-Bacharach type theorems, can be found in EGH].

Now let us describe the results of the paper and its structure in some detail. The paper is divided in two sections, including the Introduction.

Our main results of are: Theorem 2.1, Theorem 2.2, and Corollary 2.4. Here is a summary of their claims. Any variety $X \subset \mathbb{P}^{n}$ that is the zero set of homogeneous polynomials of degrees $\leq d$ and contains a supra-simplicial set $\mathrm{A}$ of nodes of a given $d^{\{n\}}$-cage $\mathrm{K} \subset \mathbb{P}^{n}$ contains all the nodes of $\mathrm{K}$. Such $X$ is a complete intersection of the multi-degree $(\underbrace{d, \ldots, d}_{s})$, where $s=\operatorname{codim}\left(X, \mathbb{P}^{n}\right)$. Moreover, $X$ is smooth in the vicinity of the node set $\mathrm{N}$. The variety $X$ is completely determined by $\mathrm{A}$ and the tangent to $X$ space $\tau_{p}$ at any of the nodes $p$. Conversely, any subspace $\tau_{p} \subset T_{p}\left(\mathbb{P}^{n}\right)$ of codimension $s$, where $p \in \mathrm{N}$, with the help of A, produces such a variety $X$.

In all the figures, we restrict ourselves to depictions of cages in the space $\mathbb{R}^{3}$. Most of the figures are produced with the help of the Graphing Calculator application. In the figures, for technical reason, the nodes of the cages are invisible. Although the images depict real surfaces in only $3^{\{3\}}$ - and $4^{\{3\}}$-cages, the entire exhibition looks surprisingly rich.

We tried to make this text friendly to readers who, as the author himself, are not practitioners of Algebraic Geometry but who may enjoy a visit to the small zoo of varieties in cages, a microcosmos of the old fashion Italian style Algebraic Geometry.

\section{A Multidimensional Zoo}

As a default, we choose the base field $\mathbb{A}$ to be the field of real or complex numbers. In the notations below, we do not emphasize the dependence of our constructions on the choice of a base field.

Let $\mathcal{L}_{j}$ be a degree $d$ homogeneous polynomial whose zero set is the union of $d$ hyperplanes of a particular color $\alpha_{j}\left(\mathcal{L}_{j}\right.$ is a product of $d$ linear forms). Since $\operatorname{deg}\left(\mathcal{L}_{j}\right)=d$, Bézout's Theorem implies that the solution set $\mathrm{N}$ of the system $\left\{\mathcal{L}_{j}=0\right\}_{j \in[1, n]}$ consists of $d^{n}$ points at most, provided that $\mathrm{N}$ is finite. Thus Definition 1.1 implies that each node $p \in \mathrm{N}$ of the cage belongs to a single hyperplane of a given color and the hyperplanes of 


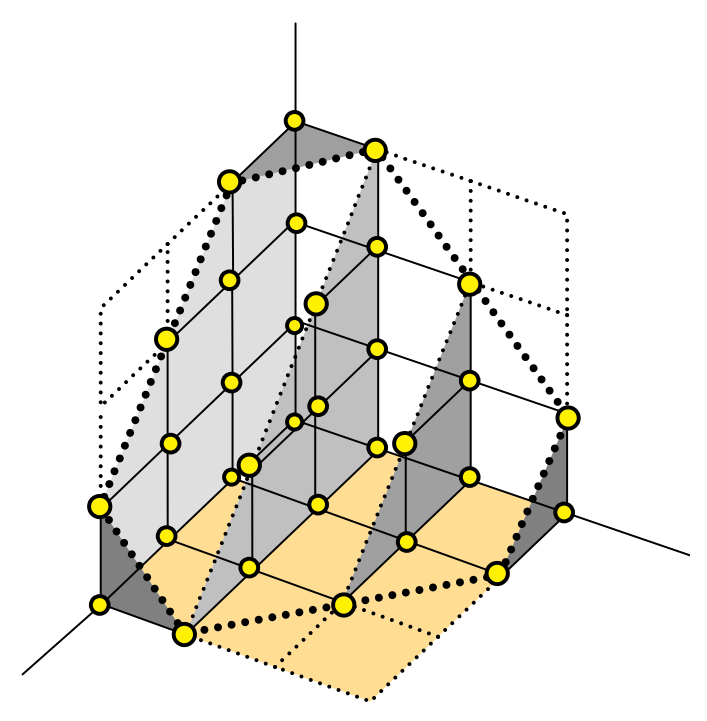

Figure 2. A supra-simplicial set of nodes in a $4^{\{3\}}$-cage

distinct colors are in general position at $p$, and thus in the ambient $n$-space. It follows that the node locus $\mathrm{N} \subset \mathbb{P}^{n}$ is a 0 -dimensional complete intersection of degree $d^{n}$.

Example 2.1. Consider the complex Fermat curve $\mathcal{F} \subset \mathbb{C P}^{2}$, given by the equation $\left\{\tilde{x}^{d}+\tilde{y}^{d}=\tilde{z}^{d}\right\}$ in the homogeneous coordinates $[\tilde{x}: \tilde{y}: \tilde{z}]$. In the affine coordinates $(x, y)=$ $(\tilde{x} / \tilde{z}, \tilde{y} / \tilde{z})$, its equation may be written as $x^{d}+y^{d}=1$, or as $\prod_{\xi}(x-\xi)+\prod_{\eta}(y-\eta)=0$, where $\xi, \eta$ run over the set of complex $d$-roots $\{\sqrt[d]{-1 / 2}\}$. Therefore $\mathcal{F}$ passes trough the nodes of the $d \times d$-cage $\mathrm{K}:=\left(\bigcup_{\xi}\{x=\xi\}\right) \cup\left(\bigcup_{\eta}\{y=\eta\}\right) \subset \mathbb{C}^{2}$.

Let $\mathbf{I}^{n}(d)$ be the subset $\left\{I=\left(i_{1}, i_{2}, \ldots, i_{n}\right)\right\}$ of the lattice $\mathbb{Z}_{+}^{n}$, such that each $i_{j} \in[1, d]$. So $\mathbf{I}^{n}(d)$ is a $n$-dimensional "cube" of the size $d$. By definition, $\|I\|=\sum_{j=1}^{n} i_{j}$.

If we introduce some order among the hyperplanes of the same color $\alpha_{j}(j=1, \ldots, n)$, then each node $p_{I}$ of $\mathrm{K}$ will be marked with a unique multi-index $I \in \mathbf{I}^{n}(d)$.

Definition 2.1. A set of nodes $\mathrm{T}$ from $d^{\{n\}}$-cage $\mathrm{K}$ is called simplicial if, with respect to some orderings of the hyperplanes in each group, it is comprised of the nodes $\left\{p_{I}\right\}_{I \in \mathbf{I}^{n}(d)}$, subject to the constraints $\|I\| \leq d+1$.

A set of nodes $\mathrm{A}$ from a cage $\mathrm{K}$ is called supra-simplicial if, with respect to some orderings of the hyperplanes in each group, it is comprised of the nodes $\left\{p_{I}\right\}_{I \in \mathbf{I}^{n}(d)}$, subject to the constraints $\|I\| \leq d+2$. (see Figure 2, where the grid corner is located at $(1,1,1)$ ). 
Example 2.2. For $d=2$, the $2^{\{n\}}$-cage is modeled after the union of the hyperplanes in $\mathbb{R}^{n}$ that extend the faces of a $n$-cube. The cardinality of the node locus $\mathrm{N}$ is $2^{n}$, the cardinality of the simplicial set $\mathrm{T}$ is $n+1$, while the cardinality of the supra-simplicial set $\mathrm{A}$ is $C_{n}^{2}+n+1=\frac{1}{2}\left(n^{2}+n+2\right)$.

Example 2.3. A famous example of a $K 3$-surface is given by the equation $\left\{y_{0}^{4}+y_{1}^{4}+\right.$ $\left.y_{2}^{4}+y_{3}^{4}=0\right\}$ in $\mathbb{C P}^{3}$, or by the equation $\left\{x_{1}^{4}+x_{2}^{4}+x_{3}^{4}+1=0\right\}$ in $\mathbb{C}^{3}$. Using the partition $\{1=1 / 3+1 / 3+1 / 3\}$, the latter equation may be written in the form

$$
\prod_{\alpha}\left(x_{1}-\alpha\right)+\prod_{\beta}\left(x_{2}-\beta\right)+\prod_{\gamma}\left(x_{3}-\gamma\right)=0,
$$

where $\alpha, \beta, \gamma$ each runs over the four complex roots of the equation $\left\{z^{4}=-1 / 3\right\}$. Therefore, the $K 3$-surface contains all the 64 nodes of a $4^{\{3\}}$-cage $\mathrm{K}$, defined by the equations

$$
\left\{\prod_{\alpha}\left(y_{1}-\alpha \cdot y_{0}\right)=0\right\} \bigcup\left\{\prod_{\beta}\left(y_{2}-\beta \cdot y_{0}\right)=0\right\} \bigcup\left\{\prod_{\gamma}\left(y_{3}-\gamma \cdot y_{0}\right)=0\right\} .
$$

In fact, the $K 3$-surface is nailed to the notes of a 2-dimensional variety of cages, produced in similar ways by writing down 1 as a sum of three complex numbers, all different from 0 . The previous construction was based on the composition $\{1=1 / 3+1 / 3+1 / 3\}$.

We notice that the nodes of this cage $\mathrm{K}$ are "invisible" in $\mathbb{R P}^{3}$.

The permutation group $\mathrm{S}_{4}$ of order 24 acts on $\mathbb{C P}^{3}$ by permuting the coordinates $\left(y_{0}, y_{1}, y_{2}, y_{3}\right)$. Under this $\mathbf{S}_{4}$-action, this surface is invariant. In contrast, the cage $\mathbf{K}$ is invariant only under the $\mathrm{S}_{3}$-action that permutes the coordinates $\left(y_{1}, y_{2}, y_{3}\right)$. (This action does not preserve the colors of the cage!) Thus, using the $\mathrm{S}_{4}$-action on $\mathrm{K}$, the $K 3$-surface contains the nodes of at least four distinct $4^{\{3\}}$-cages in $\mathbb{C P}^{3}$.

Example 2.4. Recall a remarkable Cayley-Salmon Theorem [C1]: any smooth complex cubic surface $X$ contains exactly 27 lines. If $X \subset \mathbb{C P}^{3}$ is given by the equation $\left\{z_{0}^{3}+z_{1}^{3}+\right.$ $\left.z_{2}^{3}+z_{3}^{3}=0\right\}$ (this surface is called Fermat cubic surface), then putting $\omega:=e^{2 \pi \mathbf{i} / 3}$, each of these 27 lines is given by 2 linear constraints (see [M], Corollary (8.20)):

$$
\begin{aligned}
& \left\{z_{0}+\omega^{i} z_{1}=0, z_{2}+\omega^{j} z_{3}=0\right\}, \quad i, j \in[0,2], \\
& \left\{z_{0}+\omega^{i} z_{2}=0, z_{1}+\omega^{j} z_{3}=0\right\}, \quad i, j \in[0,2], \\
& \left\{z_{0}+\omega^{i} z_{3}=0, z_{1}+\omega^{j} z_{2}=0\right\}, \quad i, j \in[0,2] .
\end{aligned}
$$

As in the previous examples, using the composition $\{1=1 / 3+1 / 3+1 / 3\}$, we notice that $X$ is inscribed in a $3^{\{3\}}$-cage $\mathrm{K}$, given by the formula

$$
\bigcup_{j=1}^{3}\left\{\prod_{k=0}^{2}\left(z_{j}+\frac{1}{\sqrt[3]{3}} \omega^{k} z_{0}\right)=0\right\} .
$$

As in Example 2.3, there exists a 2-parameter family of cages in which $X$ is inscribed (it corresponds to different ways one can represent 1 as a sum of three non-vanishing complex numbers). 
The symmetric group $\mathrm{S}_{4}$ acts on the Fermat surface $X$ by permuting the coordinates in $\mathbb{C P}^{3}$. This action must preserve the configuration of 27 lines in $X$ since these lines are the only ones residing in $X$. The subgroup $\mathrm{S}_{3} \subset \mathrm{S}_{4}$ that permutes the coordinates $\left(z_{1}, z_{2}, z_{3}\right)$ evidently preserves the cage $\mathrm{K}$, but not its colors. Thus $X$ contains the nodes of at least 4 distinct cages in $\mathbb{C P}^{3}$, obtained from $\mathrm{K}$ by the $\mathrm{S}_{4}$-action.

Consider the 27 lines, contained the $3^{\{3\}}$-cage $\mathrm{K}$, where two planes of distinct colors intersect (this locus is the "1-skeleton" of K), and compare them with the 27 lines on a smooth cubic surface $X$ (see $[\mathrm{H}]$, Chapter V, Section 4, for the explicit description of the configuration the 27 lines on $X$ ).

Question 2.1. For a smooth complex cubic surface $X \subset \mathbb{C P}^{3}$ that contains all the nodes of a given $3^{\{3\}}$-cage $\mathrm{K}$, how to describe in terms of $\mathrm{K}$ the pattern of 27 lines that belong to $X$ ? Is there anything special about the locus where the 27 lines in $X$ hit the nine planes that form the cage?

Perhaps, within the family of cubic surfaces $X$ that are inscribed in $\mathrm{K}$, the 27 bicolored lines of the cage are "the limits" of 27 lines on $X$, as $X$ degenerates into the completely reducible variety of 3 planes of a particular color?

By examining the diagonal lines in the Pascal Triangle, we get the following useful combinatorial fact.

Lemma 2.1. Each simplicial set of nodes $\mathrm{T}$ in a $d^{\{n\}}$-cage is of the cardinality $C_{d+n-1}^{n}$.

Each supra-simplicial set of nodes $\mathrm{A}$ in a $d^{\{n\}}{ }_{-}$cage is of cardinality $C_{d+n}^{n}-n$.

Let $H_{j, i}$ be the $i$-th hyperplane of the color $\alpha_{j}$, and let $L_{j, i}$ be a homogeneous linear polynomial in the coordinates $\left(y_{0}, y_{1}, \ldots, y_{n}\right)$ on the space $\mathbb{A}^{n+1}$ that defines $H_{j, i}$. Each $L_{j, i}$ is determined, up to proportionality, by $H_{j, i}$. In what follows, we fix particular linear forms $\left\{L_{j, i}\right\}_{i, j}$. Put $\mathcal{L}_{j}:=\prod_{i \in[1, d]} L_{j, i}$.

For any nonzero vector $\vec{\lambda}=\left(\lambda_{1}, \ldots, \lambda_{n}\right) \in \mathbb{A}^{n}$, we consider the homogeneous polynomial of degree $d$

$$
\mathcal{P}_{\mathrm{K}, \vec{\lambda}}:=\sum_{j \in[1, n]} \lambda_{j} \cdot \mathcal{L}_{j}
$$

Evidently, each polynomial $\mathcal{P}_{\mathrm{K}, \vec{\lambda}}$ vanishes at all the nodes of the cage $\mathrm{K}$.

Theorem 2.1. Consider a subvariety $V \subset \mathbb{P}^{n}$, given by one or several homogeneous polynomial equations of degrees $\leq d$.

- If $V$ contains all the nodes from a supra-simplicial set $\mathrm{A}$ of a $d^{\{n\}}$-cage $\mathrm{K} \subset \mathbb{P}^{n}$, then $V$ contains all $d^{n}$ nodes of the cage. Moreover, any such variety $V$ is given by polynomial equations of the form $\left\{\mathcal{P}_{\mathrm{K}, \vec{\lambda}}=0\right\}_{\vec{\lambda}}$ for an appropriate choice of vectors $\vec{\lambda}$ (see 2.2)).

- In contrast, no such variety $V$ contains all the nodes from a simplicial set $\tilde{\mathbf{T}}$ of any $(d+1)^{\{n\}}$-cage $\tilde{\mathrm{K}} \subset \mathbb{P}^{n}$. 


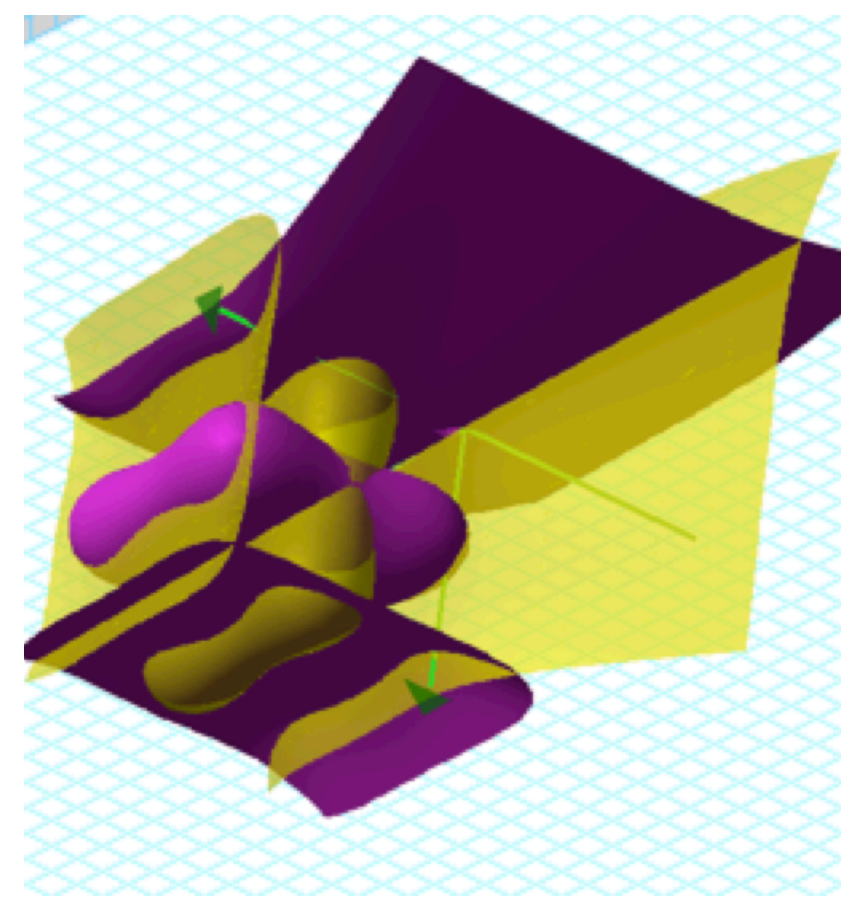

Figure 3. Two cubic surfaces, each passing through the nodes of a $3^{\{3\}}$-cage $\mathrm{K} \subset \mathbb{R}^{3}$. Note the curve $C \subset \mathbb{R}^{3}$ of the multi-degree $(3,3)$, where the two surfaces (of two colors) intersect. $C$ also contains all the 27 nodes of K.

Proof. As in the case of encaged plane curves [K], the argument is based on a combinatorial similarity between the Newton's diagram of a generic polynomial of degree $d$ in $n$ variables and a simplicial set $\tilde{T}$ of nodes of any $(d+1)^{\{n\}}$-cage. Also the cardinality of such a Newton's diagram exceeds the cardinality of a supra-simplicial set $\mathrm{A}$ of nodes of a $d^{\{n\}}$-cage $\mathrm{K}$ by $n$. In other words, the dimension of the variety of hypersurfaces of degree $d$ in the space $\mathbb{P}^{n}$ exceeds \#A by $n-1$. Indeed, the monomials in the affine variables $x_{1}, \ldots, x_{n}$ of degree $\leq d$ (equivalently, the homogeneous monomials in the variables $y_{0}, \ldots, y_{n}$ of degree $d$ ) are in oneto-one correspondence with the set B none-negative integral $n$-tuples $I \in \mathbb{Z}^{n}$, subject to the inequality $\|I\| \leq d$. At the same time, the nodes $\left\{p_{I}\right\}$ of an supra-simplicial set A satisfy the 


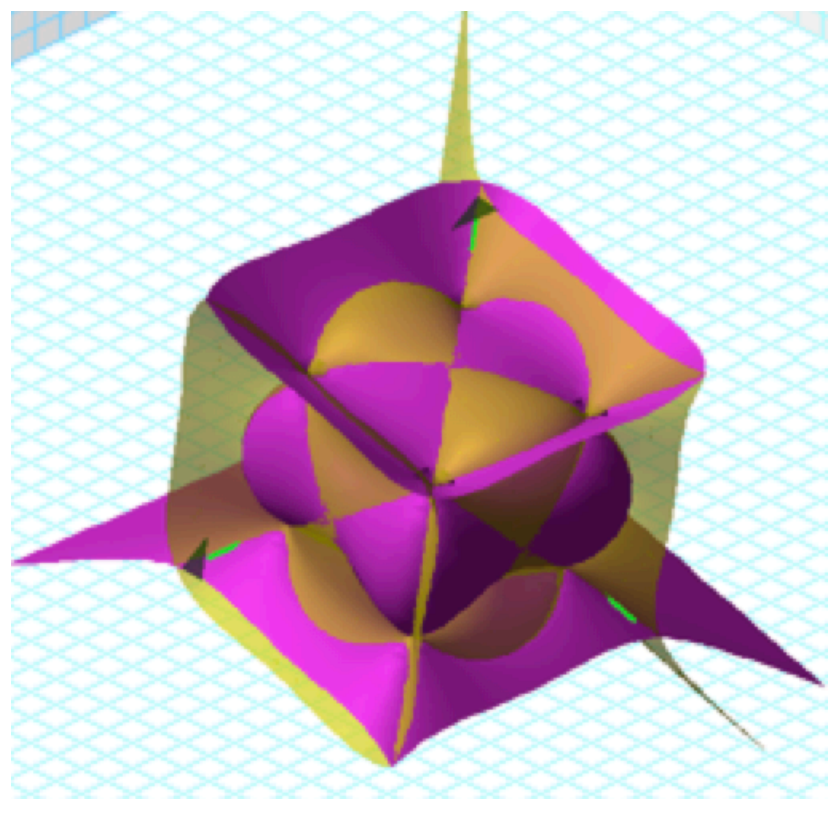

FiguRE 4. Another pair of cubic surfaces, passing trough the 27 nodes of the same cage $\mathrm{K}$, as in Figure 3. Again, the "bicolored" intersection locus $C$ of the two surfaces contains all 27 nodes of $\mathrm{K}$.

inequality $\|I\| \leq d+2$ together with $\left\{1 \leq i_{s} \leq d\right\}_{s \in[1, n]}$. Shifting by the vector $(-1, \ldots,-1)$ embeds $\mathrm{A}$ into $\mathrm{B}$ so that only the $n$ corners $(d, 0, \ldots, 0),(0, d, \ldots, 0), \ldots(0,0, \ldots, d)$ of the Newton diagram remain outside of the shifted A. Finally, proportional polynomials define the same hypersurface.

The following proof is recursive in nature. The induction is carried in $n$, the dimension of the cage. We assume that the first assertion of the theorem is valid for all $d^{\{k\}}$-cages of any size $d$ in spaces of dimension $k<n$, and the second assertion is valid for all cages of any size $d+1$ in spaces of dimension $k<n$.

Our argument relies on slicing $\mathrm{K} \supset \mathrm{A}$ by the hyperplanes $\left\{H_{1, i}=0\right\}_{i \in[1, d]}$ of the first color $\alpha_{1}$, thus reducing the argument to families of cages in $(n-1)$-dimensional affine or 


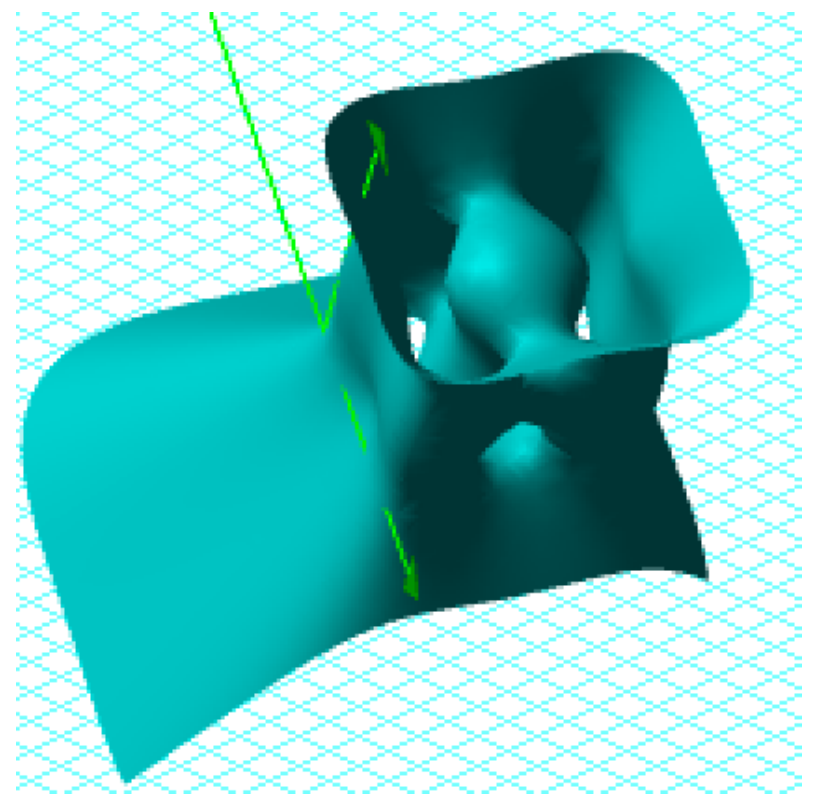

FiguRE 5. A surface of degree 4 , passing trough 64 nodes of a $\mathrm{S}_{3}$-symmetric $4^{\{3\}}$-cage $\mathrm{K} \subset \mathbb{R}^{3}$. Although this surface is not compact in $\mathbb{R}^{3}$, there are compact real surfaces of degree 4 that pass trough the nodes of $\mathrm{K}$.

projective spaces. This leads to a "Fubini-type cage theorem" in the spirit of [GHS2] (see Figure 2 for guidance). 
For any integer $s \in[1, d-1]$, we consider the $(d-s+1)^{\{n-1\}}$ sub-cage $\mathrm{K}^{[s]} \subset \mathrm{K} \cap H_{1, s}$, formed by the hyperplanes

$$
H_{1, s} \bigcap\left(\bigcup_{j \in[2, n], i \in[1, d-s+1]} H_{j, i}\right)
$$

in $H_{1, s} \approx \mathbb{P}^{n-1}$. In the hyperplane $H_{1, s}$, the cage $\mathrm{K}^{[s]}$ is given by the equation

$$
\left\{\mathcal{L} \mathcal{L}^{[s]}:=\prod_{j \in[2, n],} \prod_{i \in[1, d-s+1]} L_{j, i}=0\right\} .
$$

We denote by $\mathrm{T}^{[s]}$ the simplicial set of nodes in $\mathrm{T} \cap \mathrm{K}^{[s]}$ and by $\mathrm{A}^{[s]}$ - the set of nodes from the supra-simplicial set $A \cap K^{[s]}$. Note that the set $T^{[s]}$ can serve as a simplicial set and $A^{[s]}$ - as a supra-simplicial set for the cage $K^{[s]}$.

We start with a given homogeneous degree $d$ polynomial $P$ in the projective coordinates $\left[y_{0}: y_{1}: \ldots y_{n}\right]$, which vanishes at all the nodes of a supra-simplicial set $\mathrm{A}$ of a $d^{\{n\}}$-cage $\mathrm{K} \subset \mathbb{P}^{n}$.

Consider the restriction of $P$ to the first hyperplane $H_{1,1}$ of the color $\alpha_{1}$. Then $P$ vanishes at the supra-simplicial set $\mathrm{A}^{[1]}:=\mathrm{A} \cap H_{1,1}$ of the induced $d^{\{n-1\}}$-cage $\mathrm{K}^{[1]}:=\mathrm{K} \cap H_{1,1}$, the zero set of the polynomial $\mathcal{L}_{2} \cdot \mathcal{L}_{3} \cdot \ldots \cdot \mathcal{L}_{n}$ in $H_{1,1}$. By induction on $n$, the restriction $\left.P\right|_{H_{1,1}}$ must be of the form $\mathcal{P}_{1}:=\sum_{j \in[2, n]} \lambda_{j}^{[1]} \cdot \mathcal{L}_{j}$ (being restricted to $H_{1,1}$ ) for some choice of the coefficients $\lambda_{2}^{[1]}, \ldots \lambda_{n}^{[1]}$. For this special choice of $\left(\lambda_{2}^{[1]}, \ldots \lambda_{n}^{[1]}\right)$, the difference $P-\mathcal{P}_{1}$ is identically zero on $H_{1,1}$. If a homogeneous polynomial $R$ vanishes on a hyperplane, given by a homogeneous linear polynomial $L$, then $R$ is divisible by $L$. Indeed, by a linear change of the homogeneous coordinates, we may choose $L$ as the first new variable and write down $R$ as $L \cdot Q+S$, where $S$ is a homogeneous polynomial that depends only on the rest of the new variables. Since $S=\left.S\right|_{\{L=0\}}=0$, the polynomial $S=0$ identically, and $R=L \cdot Q$.

Therefore $P-\mathcal{P}_{1}$ is divisible by the liner polynomial $L_{1,1}$. So $P=\mathcal{P}_{1}+L_{1,1} \cdot P_{1}$, where $P_{1}$ is a homogeneous polynomial of degree $d-1$.

Next, we consider the restrictions of $P$ and $P_{1}$ to the hyperplane $H_{1,2}=\left\{L_{1,2}=0\right\}$ of color $\alpha_{1}$. Since both $P$ and $\mathcal{P}_{1}$ vanish at the set $\mathrm{A} \cap H_{1,2}$ and, by Definition $1.1, L_{1,1} \neq 0$ at the points of $\mathrm{A} \cap H_{1,2}$, we conclude that $P_{1}$ (of degree $d-1$ ) must vanish at the set $\mathrm{A} \cap H_{1,2}$ as well. Note that $\mathrm{A} \cap H_{1,2}=\mathrm{A}^{[2]}$ is a simplicial set for the induced $d^{\{n-1\}}$-cage $\mathrm{K}^{[2]} \subset \mathrm{K} \cap H_{1,2}$. So by induction, any homogeneous polynomial of degree $d-1$ that vanishes at a simplicial set $\mathrm{A}^{[2]}$ of the $d^{\{n-1\}}$-cage $\mathrm{K}^{[2]}$ must vanish at $H_{1,2}$. Hence $P_{1}=L_{1,2} \cdot P_{2}$ for some homogeneous polynomial $P_{2}$ of degree $d-2$. So we get $P=\mathcal{P}_{1}+L_{1,1} \cdot L_{1,2} \cdot P_{2}$.

Similarly, we argue that of $P_{2}$ of degree $d-2$ vanishes on the simplicial set $\mathrm{A}^{[3]} \subset \mathrm{A} \cap H_{1,3}$ of the $(d-1)^{\{n-1\}}$-cage $\mathrm{A}^{[3]}$. Therefore $\left.P_{2}\right|_{H_{1,3}}$ is zero, and $P_{2}=L_{1,3} \cdot P_{3}$ for a homogeneous polynomial $P_{3}$ of degree $d-3$. As a result, $P=\mathcal{P}_{1}+L_{1,1} \cdot L_{1,2} \cdot L_{1,3} \cdot P_{3}$.

Continuing this reasoning, we get eventually

$$
P=\mathcal{P}_{1}+\lambda\left(L_{1,1} \cdot L_{1,2} \cdots L_{1, n}\right)=\sum_{j \in[2, n]} \lambda_{j}^{[1]} \cdot \mathcal{L}_{j}+\lambda \mathcal{L}_{1},
$$


where $\lambda$ is a constant. Therefore, $P=\lambda \cdot \mathcal{L}_{1}+\sum_{j \in[2, n]} \lambda_{j}^{[1]} \cdot \mathcal{L}_{j}$ is of the form $\mathcal{P}_{\mathrm{K}, \vec{\lambda}}$ and must vanish at every node of the $d^{\{n\}}$-cage $\mathrm{K} \subset \mathbb{P}^{n}$.

By a similar reasoning, we will validate the second claim of the theorem. So we take any polynomial $P$ of degree $d$ that vanishes at a simplicial set $\tilde{\mathrm{T}}$ of a $(d+1)^{\{n\}}$-cage $\tilde{\mathrm{K}} \subset \mathbb{P}^{n}$. As before, we slice $\tilde{\mathrm{K}}$ by the hyperplanes $\left\{H_{1, s}\right\}_{i \in[1, d+1]}$ of the color $\alpha_{1}$. Now all the slices $\tilde{\mathrm{T}}^{[s]}$ (including the first one) are simplicial sets on $\tilde{\mathrm{K}}^{[s]}$. The latter locus $\tilde{\mathrm{K}}^{[s]}$ is given by the equations

$$
\left\{\tilde{\mathcal{L L}}^{[s]}:=\prod_{j \in[2, n],} \prod_{i \in[1, d-s+2]} L_{j, i}=0\right\} .
$$

Since $P$ vanishes at $\tilde{T}^{[1]}$, by the induction hypotheses, $\left.P\right|_{H_{1,1}}=0$. This implies that $P=L_{1,1} \cdot P_{1}$, where $P_{1}$ is a homogeneous polynomial of degree $d_{1}$. The set $\tilde{\mathrm{T}}^{[2]}$ is simplicial in the cage $d^{\{n-1\}}$-cage. Since $\left.L_{1,1}\right|_{\tilde{T}^{[2]}} \neq 0$, we get that $P_{1}$ must vanish at the nodes from $\tilde{\mathrm{T}}^{[2]}$. By induction, this implies that $\left.P_{1}\right|_{H_{1,1}}=0$ and thus is divisible by $L_{1,1}$. So $P=L_{1,1} \cdot L_{1,2} \cdot P_{2}$ for a homogeneous polynomial $P_{2}$ of degree $d-2$. Continuing this process, we get $P=L_{1,1} \cdot L_{1,2} \cdot \ldots, L_{1, d} \cdot \lambda$ must vanish at the unique node of the set $\tilde{\mathrm{T}}^{[d+1]}$. This forces $\lambda=0$, and so $P$ is identically zero.

Finally, the validity of the basis of induction " $n=1$ " is obvious for univariate polynomials of any degree $d$. In fact, Theorem 2.1 has been proven in $\mathrm{K}$ for $n=2$.

Since the varieties $V$ we consider in the theorem are defined by polynomials of degrees $\leq d$, the claim follows.

Remark 2.1. Note that the assumption that $A$ is supra-simplicial set in Theorem 1.1 is essential: not any subset of nodes of the cardinality \#A from a $d^{\{n\}}$-cage imposes independent relations on the set of homogeneous polynomials of degree $d$ in $n+1$ variables!

For example, in a $4 \times 4$-cage, $\# \mathrm{~A}=13$. However, if $\mathrm{B}$ is the complement to the set of four nodes $\mathrm{C}:=\left\{p_{42}, p_{43}, p_{44}\right\}$, then not every curve of degree 4 that contains $\mathrm{B}$ will contain C. In fact, B is contained in the union of three red and one blue lines from the cage; they all miss $\mathrm{C}$.

Example 2.5. Consider any curve $C$ in $\mathbb{P}^{3}$, given by homogeneous polynomial equations of degree $\leq 3$ (typically, $C$ is of degree 9 ). If $C$ passes through 17 nodes of a supra-simplicial set $A$ of nodes of a $3^{\{3\}}$-cage, then it passes through all the 27 nodes of the cage.

A similar conclusion holds for any surface of degree 3 in $\mathbb{P}^{3}$ that passes through the 17 nodes from $A$.

Corollary 2.1. Consider a subvariety $V \subset \mathbb{P}^{n}$, given by one or several homogeneous polynomial equations of degrees $\leq d$. If $V$ contains all the nodes from a supra-simplicial set $\mathrm{A}$ (of cardinality $C_{d+n}^{n}-n$ ) in a $d^{\{n\}}$-cage $\mathrm{K} \subset \mathbb{P}^{n}$, then all the polynomials that define $V$ are exactly of degree $d$.

Proof. By Theorem 2.1, if a homogeneous polynomial $P$ of degree less than $d$, which vanishes at $V$, also vanishes at the simplicial set $\mathrm{T} \subset \mathrm{A}$ of the $d^{\{n\}}$-cage $\mathrm{K}$, then $P=0$ identically. Thus $\operatorname{deg} P=d$, provided that $P$ is nontrivial. 
In this paper, we will use the following "working definition" of complete intersections.

Definition 2.2. A purely $(n-k)$-dimensional Zariski closed subset $V \subset \mathbb{P}^{n}$ over a field $\mathbb{A}$ of characteristic zero is a complete intersection of the type $\left(d_{1}, \ldots, d_{k}\right)$, if $V$ is an intersection of hypersurfaces $H_{1}, \ldots, H_{k}$ in $\mathbb{P}^{n}$ of degrees $d_{1}, \ldots, d_{k}$ such that, at a generic (nonsingular) point $x$ of each component of $V$, the tangent hyperspaces $\left\{T_{x} H_{j}\right\}_{j \in[1, k]}$ are in general position in the ambient tangent space $T_{x} \mathbb{P}^{n}$.

The next proposition was stated by the reviewer of this paper as a natural generalization of some claims from our Theorem 2.2. It puts this theorem in a larger context.

Proposition 2.1. Suppose that $W \subset \mathbb{P}^{n}$ is a smooth complete intersection of $r \leq n$ hypersurfaces of degree $d$ and that $V \supset W$ is an algebraic subset of $\mathbb{P}^{n}$ that is (set-theoretically) an intersection of hypersurfaces of degree $d$. Then is $V$ is a complete intersection of hypersurfaces of degree $d$, and $V$ is smooth along $W$.

Proof. Let homogeneous polynomials $g_{1}, \ldots, g_{r} \in \mathbb{A}\left[x_{0}, \ldots, x_{n}\right]$ of degree $d$ be generators of the zero ideal $\mathcal{I}(W)$ of the complete intersection $W$ of codimension $r$. In particular, they are linearly independent vectors of the $d$-graded portion of the ring $\mathbb{A}\left[x_{0}, \ldots, x_{n}\right]$. Let $V$ be the zero set of homogeneous degree $d$ polynomials $f_{1}, \ldots, f_{s} \in \mathbb{A}\left[x_{0}, \ldots, x_{n}\right]$. Since $V \supset W$, each $f_{j} \in \mathcal{I}(W)$. Since $f_{j}$ is of the degree $d$, we get $f_{j}=\sum_{k} c_{k j} g_{k}$, a linear combination of $g_{k}$ 's over $\mathbb{A}$. We may choose a maximal subset of the set $\left\{f_{1}, \ldots, f_{s}\right\}$, comprised of linearly independent elements. By linear algebra, $s \leq r$. Abusing notations, we denote these new elements by $f_{1}, \ldots, f_{t}$. To show that $V$ is a complete intersection, we need to show that the codimension of $V$ in $\mathbb{P}^{n}$ is $t$. At a smooth point $p \in W$, in an affine chart that contains $p$, we replace the homogeneous polynomials $g_{1}, \ldots, g_{r}$ by their non-homogeneous representations $\tilde{g}_{1}, \ldots, \tilde{g}_{r}$ in the affine coordinates. Then the differentials $d \tilde{g}_{1}, \ldots, d \tilde{g}_{r}$ at $p$ (that span the normal cotangent to $W$ bundle) are linearly independent. Thus, by linear algebra, $d \tilde{f}_{1}, \ldots, d \tilde{f}_{t}$ are linearly independent at $p$ as well. Therefore $V$ is smooth at $p \in W$ and $\operatorname{codim}\left(V, \mathbb{P}^{n}\right)=t$.

We choose the node set $\mathrm{N} \subset \mathrm{K}$ for the role of the complete intersection $W$ from Proposition 2.1. With this choice, Proposition 2.1, being combined with Theorem 2.1. implies directly Theorem 2.2 below.

Theorem 2.2. Let $V \subset \mathbb{C P}^{n}$ be a subvariety of codimension s, given by one or several homogeneous polynomial equations of degrees $\leq d$.

If $V$ contains all the nodes from a supra-simplicial set $\mathrm{A}$ of a $d^{\{n\}}$-cage $\mathrm{K} \subset \mathbb{C P}^{n}$, then $V$ is a complete intersection of the multi-degree $(\underbrace{d, \ldots, d}_{s})$, which is smooth at each node of the cage K. Thus, $\operatorname{deg} V=d^{n-\operatorname{dim} V}$.

In turn, Theorem 2.2 forces the following obvious logical conclusion.

Corollary 2.2. If a variety $V \subset \mathbb{C P}^{n}$, given by homogeneous polynomials of degrees $\leq d$, is not a complete intersection, then it cannot be trapped in any $d^{\{n\}}$-cage in $\mathbb{P}^{n}$. 
Perhaps, Theorem 2.2 and Corollary 2.2 are valid also over the base field $\mathbb{R}$.

Example 2.6. Since the twisted cubic curve $\mathcal{C}:[s: t] \rightarrow\left[s^{3}: s^{2} t: s t^{2}: t^{3}\right]$ is not a complete intersection in $\mathbb{C P}^{3}$, by Corollary 2.2, $\mathcal{C}$ does not contain the nodes of any $3^{\{3\}_{-}}$ cage $\mathrm{K}$ in $\mathbb{C P}^{3}$, or even the nodes from a supra-simplicial set $\mathrm{A} \subset \mathrm{K}$.

Example 2.7. Despite looking diverse, all the figures in this paper depict varieties, attached to the nodes of $d^{\{n\}}$-cages $\mathrm{K}(\mathbf{Q})$ that are produced following a very simple recipe. It starts with a small set $\mathbf{Q} \subset \mathbb{A}^{n}$ of "nodes in the making" and uses the product structure in $\mathbb{A}^{n}$ as follows.

Consider $d$ points $q_{1}, \ldots, q_{d} \in \mathbb{A}^{n}$ such that, for each coordinate function $z_{j}: \mathbb{A}^{n} \rightarrow \mathbb{A}$, their $z_{j}$-coordinates are distinct. Let us denote by $\mathbb{A}(n, d)$ the space of such configurations $\mathbf{Q}:=\left(q_{1}, \ldots, q_{d}\right)$. Then each $\mathbf{Q} \in \mathbb{A}(n, d)$ produces a $d^{\{n\}}$-cage $\mathbf{K}(\mathbf{Q}) \subset \mathbb{A}^{n}$, formed by the hyperplanes $\left\{H_{j, i}:=z_{j}^{-1}\left(z_{j}\left(q_{i}\right)\right)\right\}_{j \in[1, n], i \in[1, d]}$. By Theorem 2.1 and Theorem 2.2, for any $s \leq n$, the cage $\mathrm{K}(\mathbf{Q})$ supports the family of varieties $X$ of the multi-degree $(\underbrace{d, \ldots, d}_{s})$ and dimension $n-s$ that contain the node set $\mathbf{N}(\mathbf{Q})$ of $\mathbf{K}(\mathbf{Q})$. By Theorem 2.4 below, the family is parametrized by points of the Grassmanian $\mathrm{Gr}_{\mathbb{A}}(n, n-s)$.

Over $\mathbb{C}$, we can enhance this cage construction. Consider the complex Viète map $\Sigma$ : $\mathbb{C}^{n} \rightarrow$ Sym $^{n} \mathbb{C} \approx \mathbb{C}^{n}$, given by the elementary symmetric polynomials in $z_{1}, \ldots, z_{n}$. It takes the "roots" $z_{1}, \ldots, z_{n} \in \mathbb{C}$ to the coefficients of the monic polynomial $\prod_{j=1}^{n}\left(x-z_{j}\right)$ in the variable $x$.

We denote by $\mathcal{D}$ the hypersurface in $\operatorname{Sym}^{n} \mathbb{C}$, formed by the $x$-polynomials with multiple roots. It is called the discriminant variety. Remarkably, the $\Sigma$-images of the hyperplanes $\left\{H_{j, i} \subset \mathbb{C}^{n}\right\}$ are hyperplanes, tangent to $\mathcal{D}$; moreover, the normal vector to $\Sigma\left(H_{j, i}\right)$, whose $n^{\text {th }}$ coordinate is 1 , has its $(n-1)^{\text {st }}$ coordinate equal to $z_{j}\left(q_{i}\right)$ ([K2], Corollary 6.1)!

Therefore, $\left\{\Sigma\left(H_{j, i}\right)\right\}_{j, i}$ form a new $d^{\{n\}}$-cage $\Sigma(\mathrm{K}(\mathbf{Q}))$ in Sym ${ }^{n} \mathbb{C} \approx \mathbb{C}^{n}$, whose hyperplanes are tangent to the discriminant variety $\mathcal{D}$. The nodes of $\Sigma(\mathrm{K}(\mathbf{Q}))$ reside in $\mathbb{C}^{n}$. Via the tangency property, the cage $\Sigma(\mathrm{K}(\mathbf{Q}))$ is completely determined by the configuration $\Sigma(\mathbf{Q})$ of $d$ points in $\mathbb{C}^{n} \backslash \mathcal{D}$, since any point $p \in \mathbb{C}^{n} \backslash \mathcal{D}$ belongs to exactly $n$ hyperplanes that are tangent to $\mathcal{D}[\mathrm{K} 2$. As a result, any generic (that is, of the form $\Sigma(\mathbb{C}(n, d))$ ) configuration $\mathbf{P}$ of points $p_{1}, \ldots, p_{d} \in \mathbb{C}^{n} \backslash \mathcal{D}$ produces a $d^{\{n\}}$-cage $\mathrm{K}(\mathbf{P})$ in $\mathbb{C}^{n}$, whose hyperplanes are alined with the tangent cones of $\mathcal{D}$. Again, for any $s \leq n$, the cage $\mathrm{K}(\mathbf{P})$ supports a family of complex varieties $V$ of the multi-degree $(\underbrace{d, \ldots, d}_{s})$ and dimension $n-s$ that contain the node set $\mathrm{N}(\mathbf{P})$ of $\mathrm{K}(\mathbf{P})$. By Theorem 2.4, the family is parametrized by points of the Grassmanian $\operatorname{Gr}_{\mathbb{C}}(n, n-s)$.

Thus we got an effective device for producing varieties in cages. A configuration $\mathbf{Q} \in$ $\mathbb{C}(n, d)$ or a configuration $\mathbf{P} \in \Sigma(\mathbb{C}(n, d))$, together with a choice of a $(n-s)$-dimensional affine subspace $\tau \subset \mathbb{C}^{n}$ at a point $q_{1} \in \mathbf{Q}$, or a $(n-s)$-dimensional affine subspace $\tilde{\tau} \subset \mathbb{C}^{n}$ at a point $p_{1} \in \mathbf{P}$, produce unique varieties $X(\mathbf{Q}, \tau) \subset \mathbb{C}^{n}$ and $Y(\mathbf{P}, \tilde{\tau}) \subset \mathbb{C}_{\text {coef }}^{n}$ of the dimension $n-s$ that are attached to the nodes of the two cages, respectively. 
Over the real numbers, the outcome is similar, if we consider only the chamber $\mathcal{C}$ in the space $\mathbb{R}_{\text {coef }}^{n}$ of monic real polynomials with all real roots; $\mathcal{C}$ is one of many chambers in which the real discriminant hypersurface $\mathcal{D}_{\mathbb{R}}$ divides $\mathbb{R}_{\text {coef }}^{n}$. So, over $\mathbb{R}$, the cage-generating configuration $\mathbf{P}$ must be chosen in the chamber $\mathcal{C}$.

The construction $(\mathbf{Q}, \tau) \Rightarrow X(\mathbf{Q}, \tau)$ has one pleasing property: if the configuration $\mathbf{Q}$ consists of $d$ points with all the coordinates in $\mathbb{Z}$ or $\mathbb{Q}$, then the variety $X(\mathbf{Q}, \tau)$ contains at least $d^{n}$ integral or rational points. Since the Viète map $\Sigma$ is given by elementary symmetric polynomials with integer coefficients, the same property holds for any variety $Y(\Sigma(\mathbf{Q}), \tilde{\tau}))$ that is attached to the nodes of the cage $\mathrm{K}(\Sigma(\mathbf{Q})) \subset \operatorname{Sym}^{n} \mathbb{C}$.

Remark 2.1. Recall that, thanks to the Lefschetz Hyperplane Theorem (see Corollary 7.3 and Theorem 7.4 in [Mi]), the topology of complete intersections is very special. In particular, by a theorem of Thom, the diffeomorphism type of a smooth complete intersection $X$ over $\mathbb{C}$ is determined by its dimension and its multi-degree $\left(d_{1}, \ldots, d_{k}\right)[\mathrm{LW}$. Therefore, the combinatorics of a supra-simplicial set imposes strong restrictions on the homology groups, homotopy types, and characteristic classes of the varieties inscribed in a given cage.

Let us consider a $n$-dimensional polyhedron $\mathcal{P}$ in $\mathbb{R}^{n}$, whose combinatorics is modeled after the combinatorics of a $n$-cube. The opposite faces of $\mathcal{P}$ are labeled with the same color; so the total pallet has $n$ colors. We wish to place the vertices of $\mathcal{P}$ on a given variety $V \subset \mathbb{R}^{n}$ that is defined as the zero set of several quadratic polynomials (think about $V$ as being an ellipsoid or a hyperboloid). The next corollary testifies that in order to accomplish this task, one needs to place just few vertices of $\mathcal{P}$ on $V$, the rest of the vertices will reside in $V$ automatically. Actually, the following direct corollary of Theorem 2.2 makes sense over any infinite field $\mathbb{A}$.

Recall that any complex projective variety may be given as an intersection of quadrics in an appropriate projective space [M1].

Corollary 2.3. (Varieties in the Cube Cage) Let a variety $V \subset \mathbb{P}^{n}$ be given by homogeneous polynomials of degree 2 and contains all $\frac{1}{2}\left(n^{2}+n+2\right)$ nodes of a suprasimplicial set $\mathrm{A}$ in a $2^{\{n\}}$-cage $\mathrm{K} \subset \mathbb{P}^{n}$.

Then $V$ is a complete intersection of degree $2^{s}$, where $s=n-\operatorname{dim}(V)$. Moreover, $V$ contains all $2^{n}$ nodes of $\mathrm{K}$ and is smooth in their vicinity.

Example 2.9. If a smooth curve $C \subset \mathbb{P}^{3}$ is given by two homogeneous quadratic forms and contains 7 nodes of a $2^{\{3\}}$-cage $\mathrm{K} \subset \mathbb{P}^{3}$, then it contains the $8^{\text {th }}$ node of the cage. Moreover, $C$ is a complete intersection of the multi-degree $(2,2)$. In fact, such a curve $C$ is elliptic (i.e., smooth and of genus 1).

Proposition 2.2 below is another claim, formulated by the reviewer. It frames well our Corollary 2.4.

Proposition 2.2. Suppose that $W \subset \mathbb{P}^{n}$ is a smooth complete intersection of $r \leq n$ hypersurfaces of degree $d$. Pick a point $p \in W$ and a linear subspace $\tau_{p} \subset T_{p} \mathbb{P}^{n}$ of dimension 
$\delta>n-r$ such that $\tau_{p} \supset T_{p} W$. Then there exists a unique complete intersection $V \subset \mathbb{P}^{n}$ of $n-\delta$ hypersurfaces of degree $d$ such that $V \supset W$ and $T_{p} V=\tau_{p}$.

Proof. As in the proof of Proposition 2.1, let homogeneous polynomials $g_{1}, \ldots, g_{r} \in$ $\mathbb{A}\left[x_{0}, \ldots, x_{n}\right]$ of degree $d$ be generators of the zero ideal $\mathcal{I}(W)$ of a smooth complete intersection $W$ of the codimension $r$. We denote by $L(W)$ the linear space spanned over $\mathbb{A}$ by $g_{1}, \ldots, g_{r}$. In an affine chart that contains the given point $p \in W$, we replace $g_{1}, \ldots, g_{r}$ by their expressions as polynomials $\tilde{g}_{1}, \ldots, \tilde{g}_{r}$ in the affine coordinates. Since $W$ is smooth at $p$, the differentials $d \tilde{g}_{1}, \ldots, d \tilde{g}_{r}$ at $p$ vanish exactly at the tangent subspace $T_{p} W$ of codimension $r$. We consider a linear subspace $\mu^{*}\left(\tau_{p}\right)$ of $\nu_{p}^{*}:=\operatorname{Span}_{\mathbb{A}}\left\{\left.d \tilde{g}_{1}\right|_{p}, \ldots,\left.d \tilde{g}_{r}\right|_{p}\right\}$ which consists of 1 -forms that vanish at the given vector space $\tau_{p} \supset T_{p} W$. Let $d \tilde{h}_{1}, \ldots, d \tilde{h}_{n-\delta}$ be a basis of the vector space $\mu^{*}\left(\tau_{p}\right)$. Then each $d \tilde{h}_{j}=\sum_{k} a_{j k} d \tilde{g}_{k}$ for some $a_{j k} \in \mathbb{A}$. Consider the polynomials $\left\{\tilde{h}_{j}:=\sum_{k} a_{j k} \tilde{g}_{k}\right\}_{j \in[1, n-\delta]}$. Denote by $\left\{h_{j}\right\}_{j \in[1, n-\delta]}$ their homogeneous representatives. Then we define $V$ by $n-\delta$ homogeneous equations $\left\{h_{j}=0\right\}_{j \in[1, n-\delta]}$, where $\left\{h_{j}\right\}$ are linearly independent elements in the $d$-graded portion of $\mathbb{A}\left[x_{0}, \ldots, x_{n}\right]$. By its construction, $V$ has $\tau_{p}$ for its tangent space at $p$. So its dimension is $\delta$. Therefore $V$ is a complete intersection which is smooth at $p$.

The same kind of arguments, based only on linear algebra, validates the claim that $V$ is unique among complete intersections of the multi-degree $(d, \ldots, d)$ and with $\tau_{p}$ for a tangent space at some point $p \in W$.

By taking the node set $\mathrm{N}$ for the role of the complete intersection $W$ in Proposition 2.2 and applying Theorem 2.2 , we get instantly the following claim.

Corollary 2.4. Consider a $d^{\{n\}}$-cage $\mathrm{K} \subset \mathbb{P}^{n}$ and a vector subspace $\tau_{p}$ of dimension $n-s$ in the tangent space $T_{p}\left(\mathbb{P}^{n}\right)$, where $p$ is a node of $\mathrm{K}$. Then there exists a unique complete intersection $V \subset \mathbb{P}^{n}$ of the multi-degree $(\underbrace{d, \ldots, d}_{s})$ and of dimension $n-s$ that contains all the nodes of $\mathrm{K}$ and whose tangent space $T_{p}\left(\stackrel{s}{V)}=\tau_{p}\right.$.

As a result, any supra-simplicial node set $\mathrm{A} \subset \mathrm{K}$ and $a(n-s)$-dimensional subspace $\left.\tau_{p} \subset T_{p}\left(\mathbb{P}^{n}\right), 1\right]$ where $p \in \mathrm{N}$, determines such a variety $V$ and the distribution of $(n-s)$ subspaces $\tau_{V}$ in $\left.T\left(\mathbb{P}^{n}\right)\right|_{\mathrm{N}}$ it produces. So the cage $\mathrm{K}$, with the help of the inscribed $V$ 's, defines canonically a "diagonal" embedding of Grassmanians

$$
\Delta_{\mathrm{K}}: \operatorname{Gr}_{\mathbb{A}}\left(T_{p}\left(\mathbb{P}^{n}\right), n-s\right) \longrightarrow \prod_{q \in \mathbb{N} \backslash p} \operatorname{Gr}_{\mathbb{A}}\left(T_{q}\left(\mathbb{P}^{n}\right), n-s\right) .
$$

We may give a slightly different interpretation to Corollary 2.4 by viewing the Grassmanian $\operatorname{Gr}_{\mathbb{A}}(n, n-s)$ as a moduli space of varieties in a given $d^{\{n\}}$-cage $\mathrm{K} \subset \mathbb{P}^{n}$. Consider a subvariety $\mathcal{E}(\mathrm{K}, p)$ of $\mathbb{P}^{n} \times \mathrm{Gr}_{\mathbb{A}}(n, n-s)$ which depends on the cage $\mathrm{K} \subset \mathbb{P}^{n}$ and its preferred node $p$. By definition, $\mathcal{E}(\mathrm{K}, p)=\left\{(x, \tau) \mid x \in V(\tau), \tau \in \mathrm{Gr}_{\mathbb{A}}(n, n-s)\right\}$, where $V(\tau) \subset \mathbb{P}^{n}$ is

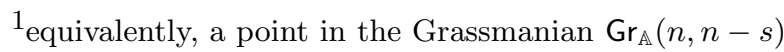


the complete intersection of dimension $n-s$ and multi-degree $(\underbrace{d, \ldots, d}_{s})$ that contains all the nodes of $\mathrm{K}$ and has $\tau$ as its tangent space $T_{p}(V(\tau))$ at $p$. Then the obvious projection $\pi: \mathbb{P}^{n} \times \mathrm{Gr}_{\mathbb{A}}(n, n-s) \rightarrow \mathrm{Gr}_{\mathbb{A}}(n, n-s)$ gives rise to a surjective regular map of algebraic varieties $\pi: \mathcal{E}(\mathrm{K}, p) \rightarrow \operatorname{Gr}_{\mathbb{A}}(n, n-s)$ whose fiber over a point $\tau \in \operatorname{Gr}_{\mathbb{A}}(n, n-s)$ is the complete intersection $V(\tau)$.

The subvarieties of the Grassmanian $\operatorname{Gr}_{\mathbb{A}}(n, n-s)$ that consist of points $\tau$ such that the fiber $\pi^{-1}(\tau)$ is singular or reducible (and thus singular) deserve a separate research.

The reader may be entertained by one special case of Corollary 2.4, where the variety $V$ is a complete intersection of the multi-degree $(\underbrace{d, \ldots, d}_{n-1})$, a curve with a given tangent line $\ell$ at one of the nodes of the cage to which $V$ is attached. In this "Cage Croquet" game, the nodes represent the gates, and the curve represents the desired trajectory of the ball, passing through all the gates. One can aim in any direction $\ell$ from any gate, and the "right trajectory" $V$ of the multi-degree $(\underbrace{d, \ldots, d}_{n-1})$ will pass through all the gates.

Let us glance now at 3-dimensional cages and the polyhedral surfaces that have their vertices among the nodes of these cages.

A tricolored polyhedral surface $\Sigma \subset \mathbb{R}^{3}$ is a surface whose faces are flat polygons, colored with three colors. We say that a vertex $v$ of $\Sigma$ is trivalent if exactly three distinctly colored faces join at $v$. A tricolored polyhedral surface is trivalent if all its vertices are. Finally, a perfect trivalent polyhedral surface is a trivalent tricolored polyhedral surface with equal number of faces, colored with each of the three colors. A surface of a cube is an example of a perfect trivalent polyhedral surface.

We notice that a generic perfect trivalent polyhedral surface with $3 d$ faces determines a $d^{\{3\}}$-cage in the space. For example, a generic union of $k$ tricolored cubes in $\mathbb{R}^{3}$ is a perfect trivalent polyhedron that gives rise to a $(2 k)^{\{3\}}$-cage in $\mathbb{R}^{3}$.

In view of these observations, Corollary 2.4 leads to the following claim.

Corollary 2.5. Let $\Sigma \subset \mathbb{R}^{3}$ be a perfect trivalent polyhedral surface with $3 d$ faces that generates a $d^{\{3\}}$-cage $\mathrm{K}_{\Sigma}$ in $\mathbb{R}^{3}$. Given a plane $\tau$ through one of vertices $v \in \Sigma$, there exists a unique affine algebraic surface $S \subset \mathbb{R}^{3}$ of degreed such that:

- all the verticies of $\Sigma$ lie on $S$ (i.e., $\Sigma$ is inscribed in $S$ ),

- $S$ contains all the nodes of the $d^{\{3\}}$-cage $\mathrm{K}_{\Sigma}$,

- $S$ is tangent to the plane $\tau$ at $v$ and is smooth in the vicinity of all verticies of $\Sigma$.

Example 2.10. The surface $\Sigma$ of a surface of a tricolored cube with three quadrangular wormholes that connect pairs of similarly colored opposite faces $(\Sigma$ is a surface of genus 3 ) has $18=6+3 \times 4$ faces ( 6 of which are not simply-connected polygons). It can be inscribed in an algebraic surface $S$ of degree $6=18 / 3$. In addition to the 32 vertices of $\Sigma$, lying on $S$, the rest of the nodes (numbering 184) of the $6^{\{3\}}$-cage $\mathrm{K}_{\Sigma}$ also belongs to $S$. Such a surface $S$ with a prescribed tangent plane $\tau$ at one vertex of $\Sigma$ is unique. 
Acknowledgments: The earlier version of this paper contained a generalization of Theorem 2.1 for cages on complex projective varieties. My proof of this generalization contained a basic irreparable mistake. I am very grateful to the reviewer who has found the mistake and came with valuable suggestions for improving the entire presentation.

\section{REFERENCES}

[B] Bacharach, I., Uber den Cayley'schen Schnittpunktsatz, Math. Ann. 26 (1886), 275-299.

[C] Cayley, A., On the Intersection of Curves, serialized in Cambridge Math. J., 25-27, and published by Cambridge University Press, Cambridge, 1889.

[C1] Cayley, A., On the triple tangent planes of surfaces of the third order, Cambridge and Dublin Math. J., 4: 118-138, (1849).

[Ch] Chasles, M., Traite de sections conique, Gauthier-Villars, Paris, 1885.

[Cl] Clebsch, A., Ueber die Anwendung der quadratischen Substitution auf die Gleichungen 5ten Grades und die geometrische Theorie des ebenen Fünfseits, Mathematische Annalen, 4 (2): 284-345, (1871).

[DGO] Davis, E. D., Geramita, A.V., and Orecchia, F., Gorenstein algebras and Cayley-Bacharach theorem, Proceedings Amer. Math. Soc. 93 (1985), 593-597.

[EGH] Eisenbud, D., Green, M., and Harris, J., Cayley-Bacharach theorems and conjectures, Bull. Amer. Math. Soc. 33 (1996), 295-324.

[F] Fulton, W., Introduction to Intersection Theory in Algebraic Geometry, Regional Conference Series in Mathematics, Number 54, American Mathematical Society, Providence, 1983.

[GHS] Geramita, A. V., Harima, T., and Shin, Y.S., Extremal point sets and Gorenstein ideals, Adv. Math $152(2000) 78-119$.

[GHS1] Geramita, A. V., Harima, T., and Shin, Y.S., An alternative to the Hilbert function for the ideal of a finite set of points in $\mathbb{P}^{n}$, Illinois J. 45 (2001) 1-23.

[GHS2] Geramita, A. V., Harima, T., and Shin, Y.S., Decompositions of the Hilbert Function of a Set of Points in $\mathbb{P}^{n}$, Canad. J. Math. 53 (2001) 925-943.

[H] Hartshorne, R., Algebraic Geometry, Springer-Verlag, New York, 1977.

[K] Katz, G., Curves in Cages: An Algebro-Geometric Zoo, The American Mathematical Monthly, 113 : 9 (2006), 777-791.

[K2] Katz, G., How Tangents Solve Algebraic Equations, or a Remarkable Geometry of Discriminant Varieties, Expositiones Math., 21 (2003), 219-261.

[LW] Libgober, A. S., Wood, J. W., Differentiable structures on complete intersections. I, Topology 21 (1982), 469-482.

[Ki] Kirwan, M. Complex Algebraic Curves, London Mathematical Society, Student Texts 23, Cambridge University Press, Cambridge, 1992.

[Kl] Klein, F., Über Flächen dritter Ordnung, Math. Ann., 6 (1873), 551-581.

[M] Mumford, D., Algebraic Geometry I, Complex Projective Varieties, Springer-Verlag, Berlin Heidelberg New York, 1976.

[M1] Mumford, D., Varieties Defined by Quadratic Equations, Centro Internazionale Matematico Estivo, Corso tenuto a Varena, Septembre 1969.

[Mi] Milnor, J., Morse Theory, Annals of Mathematical Studies, no. 51, Princeton University Press, Princeton, 1963.

[R] Ried, M. Undergraduate Algebraic Geometry, London Mathematical Society, Student Texts 12., Cambridge University Press, Cambridge, 1998.

Mit, Department of Mathematics, 77 Massachusetts Ave., Cambridge, MA 02139, U.S.A.

Email address: gabkatz@gmail.com 